\title{
Construction of Low Temperature Control Device and Management system
}

\author{
Yoshie Takewaka, Souichirou Hida, Makoto Miyauchi \\ National Institute of Technology, Kitakyushu College, 5-20-1 Shii Kokuraminami Kitakyushu, Fukuoka 802-0985, \\ Japan \\ *Corresponding Author: yoshie@kct.ac.jp
}

\begin{abstract}
IoT control has been used in many fields of engineering. But this technology is not yet general in small businesses, so it is expected to develop in future. By designing a low temperature control unit using IoT, we will clarify its advantages and problems.
\end{abstract}

Keywords: micro PC, Low Temperature Control, Feedback, IoT.

\section{Introduction}

The control technology to keep a constant temperature is carried out in many industrial fields, and the management system using IoT is used in various factories. However, these two technologies have not spread in households or small factories yet, as well they are the fields expected to develop in future.

We are intended to make a low temperature control unit which is cheaper and easy to use for small businesses by using IoT control system. This means, we are intended to conduct temperature control by transmitting data such as temperature setting or time setting to microPC (RaspberryPi) from a network terminal (PC, smartphone) and displaying current setting or temperature to a network terminal from micro PC.

\section{Low temperature control device}

The low temperature control device which we made consist of a micro PC, a solid-state relay, a temperature sensor and a heating equipment. We used a zero cross solid-state relay, that switches ON and OFF when the AC power voltage per unit time becomes $0 \mathrm{~V}$. Thus a deviation between the period of $\mathrm{AC}$ power source and the one of PWM arises if PWM period is short. In this time, we designed the device by setting one PWM period for two seconds, in order that the deviation goes within an allowable error range even if some deviation occurred.

And, we measured the temperature of the control object

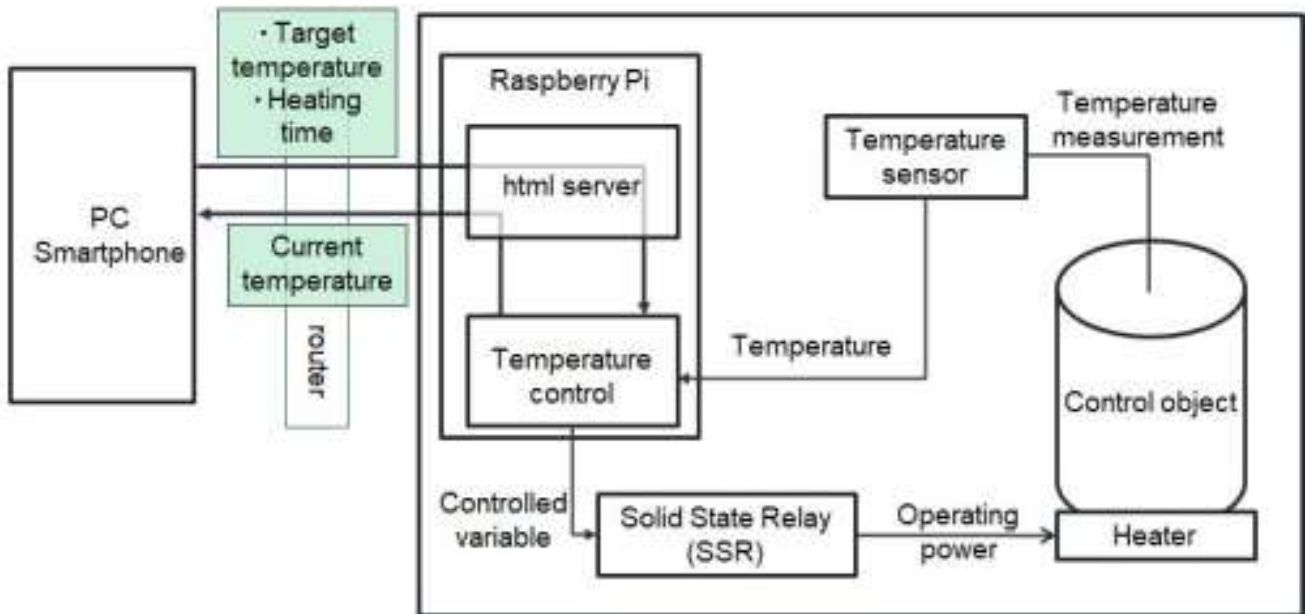

Fig. 1 Control Flow 
with a digital temperature sensor and used micro PC as a computer deciding controlled variable according to that value. And this time we adopted Raspberry Pi 3 model B as IoT apparatus because it was able to connect to the wireless Internet easily and was provided with the IO port.

We show a control flow in figure 1. First, we heat Control object in Heater, and measure the temperature with a digital temperature sensor. Next, we acquire the data in micro PC and decide controlled variable by a temperature control program. Last, we decide a PWM level based on controlled variable and control the output of Heater by operating a solid-state relay.

\section{Temperature control}

In this time, we controlled water temperature using the low temperature control device. We executed control under the following conditions:

1. to keep a constant temperature

2. to prevent overshoot

3. to use only Heater for temperature control In addition, we set various data as follows:

a target temperature was to be $60^{\circ} \mathrm{C}$, an object to be heated was $300 \mathrm{ml}$ of water, and sampling time was about 45 minutes

\subsection{On-Off control}

ON-OFF control is the control that sets the output of Heater $100 \%$ if water temperature is less than the target temperature, and that sets the output of Heater $0 \%$ if water temperature is more than the target temperature.

We show the result of measurements in figure 2. From this graph, even after the output became $0 \%$ when water temperature exceeded the target temperature, temperature continued rising and became $72^{\circ} \mathrm{C}$ and it was more than $12.0^{\circ} \mathrm{C}$ upper from the target temperature. As a result, we understood that it was necessary to limit the output gradually at an early stage in order to keep water temperature the target temperature without overshooting.

\subsection{PI control (Output 100\%)}

We designed lower $10^{\circ} \mathrm{C}$ from $60^{\circ} \mathrm{C}$ as a proportional band and configured $\mathrm{P}$ control that sets the output of heater $0 \%$ when water temperature is $60^{\circ} \mathrm{C}$ and that sets the output of heater $100 \%$ when water temperature is lower than the proportional band. When $\mathrm{P}$ control is adopted, water temperature is stabilized at the temperature that is lower than a target temperature without getting over the target

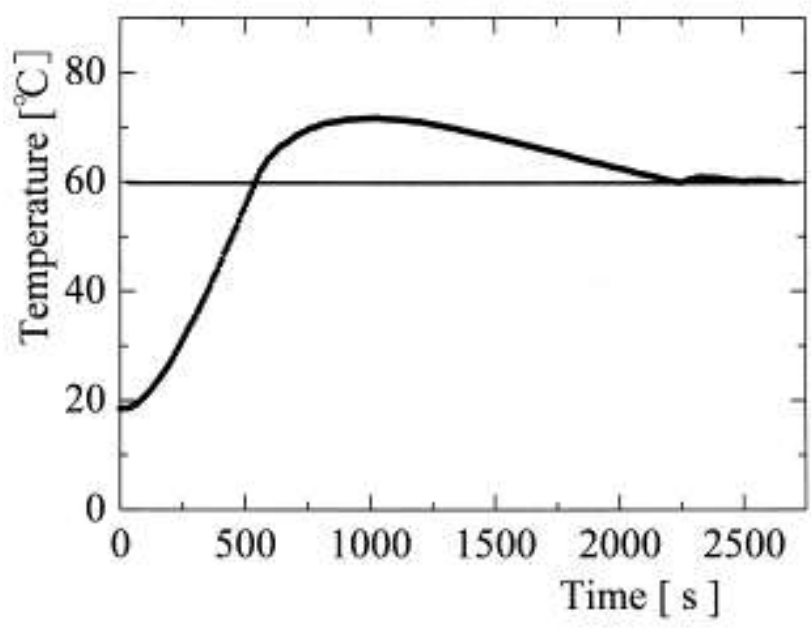

Fig.2 On-Off control

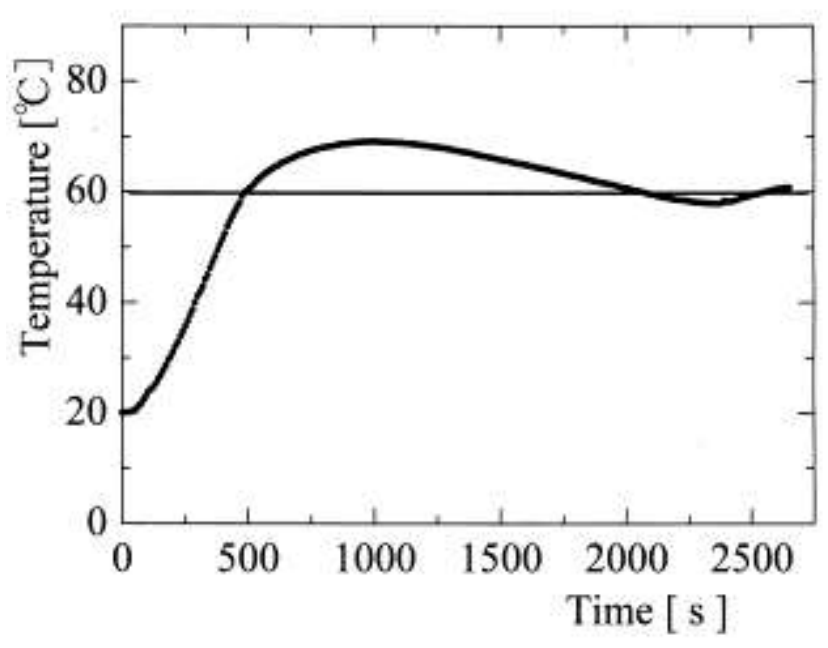

Fig.3 PI control(output:100\%)

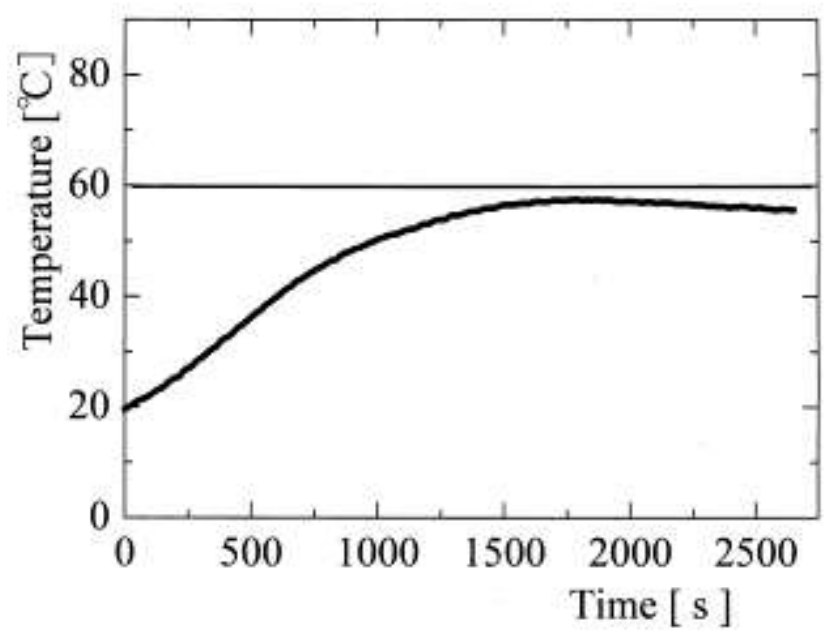

Fig.4 PI control(output:80\%) 
value if a common control apparatus is used. We generated an offset intentionally and corrected the offset afterward by I control, we were able to stabilize temperature while we suppressed occurrences of overshoot.

We show the result of measurements in figure 3. In comparison with On-Off control of figure2, we understood that overshoot $10^{\circ} \mathrm{C}$ from the target temperature were generated while some occurrence of overshoot was suppressed. The reason given for this is that the Heater continued heating the object because the Heater remained high temperature after having limited the output since the proportional band width was narrow. According to the above result, it is thought that following two are necessary:

1. to take the proportional band widely

2. to restrict the upper limit of the output

So we understood that it was necessary to make output

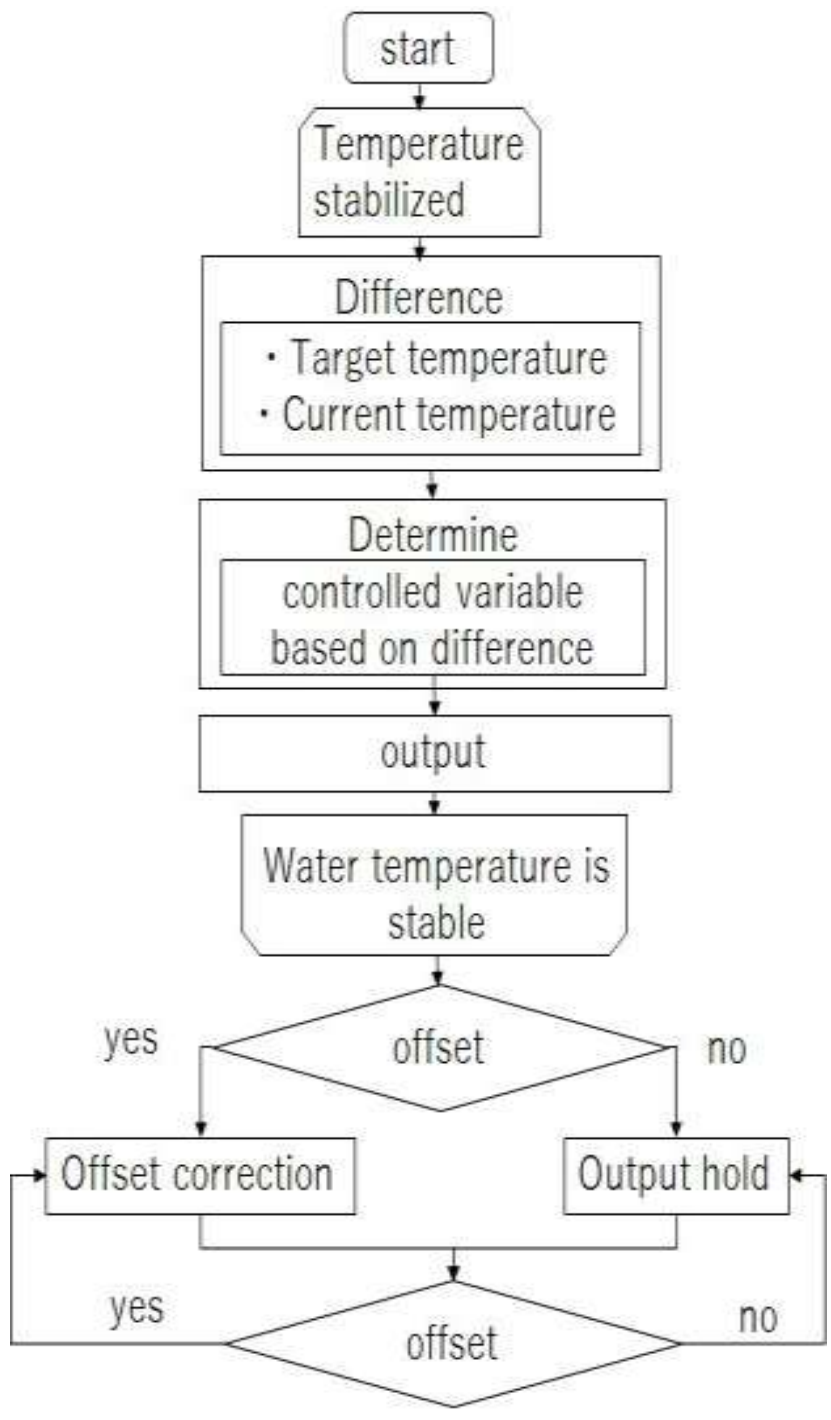

Fig.5 Control flowchart restrictions stronger

\subsection{PI control(Output 80\%)}

We set an entire range from an initial temperature to a target temperature as a proportional band of P control. In addition, a proportional band becomes narrow when an initial temperature is high, hence we set $20^{\circ} \mathrm{C}$ as an initial temperature if it exceeds $20^{\circ} \mathrm{C}$. Also we changed setting as upper limit of output $80 \%$ because it was confirmed that an overshoot might occur according to the situation when we started that experiment under the output $100 \%$.

We show the result of measurements in figure 4. It expresses that a measured temperature did not exceed a target temperature anymore. Therefore, we decided to design the experimental equipment based on this control.

We show a flow chart of the control that we designed in figure 5. We set an entire range from an initial temperature to a target temperature as a proportional band of $\mathrm{P}$ control. In addition, a proportional band becomes narrow when an initial temperature is high, hence we set $20^{\circ} \mathrm{C}$ as an initial temperature if it exceeds $20^{\circ} \mathrm{C}$. Also we changed setting as upper limit of output $80 \%$ because it was confirmed that an overshoot might occur according to the situation when we started that experiment under the output $100 \%$.

We show the result of measurements in figure 4. It expresses that a measured temperature did not exceed a target temperature anymore. Therefore, we decided to design the experimental equipment based on this control.

We show a flow chart of the control that we designed in figure 5 .

\section{Control system}

On executing the control, we developed a control program in the following environment.

Table 1. Operating environment.

\begin{tabular}{|c|l|}
\hline Text & \multicolumn{1}{|c|}{ Text } \\
\hline Control computer & RaspberryPi3 model B 2.0 \\
\hline Language & $\begin{array}{c}\text { Temperature control: python3 } \\
\text { Network: javascript,html,css }\end{array}$ \\
\hline Software & wiringPi, WebIOPi \\
\hline
\end{tabular}

Wiringpi is a software to control IO ports of RaspberryPi in Python, and WebIOPi is a software to cooperate between an $\mathrm{Html}$ site and a control program 
written in WiringPi. We made a temperature control program and a html server based on these.

In figure 6, we show the state of data exchanges, and in figure 7, we show the example of which information of the application that we made was displayed on displays of a smartphone and a PC.

First of all, a html page appears when you access to the IP address of the control computer from a network terminal. When you push the decision button after you input the preset temperature and a heating time into the page., variables in the temperature control program are changed.

And an html server calls a current temperature from the temperature control program at every fixed interval and displays it on the html page. It can display both preset information and a current temperature at the same time. When it is accessed at the same time from different terminals, current settings come to be shared at plural terminals by performing this processing.

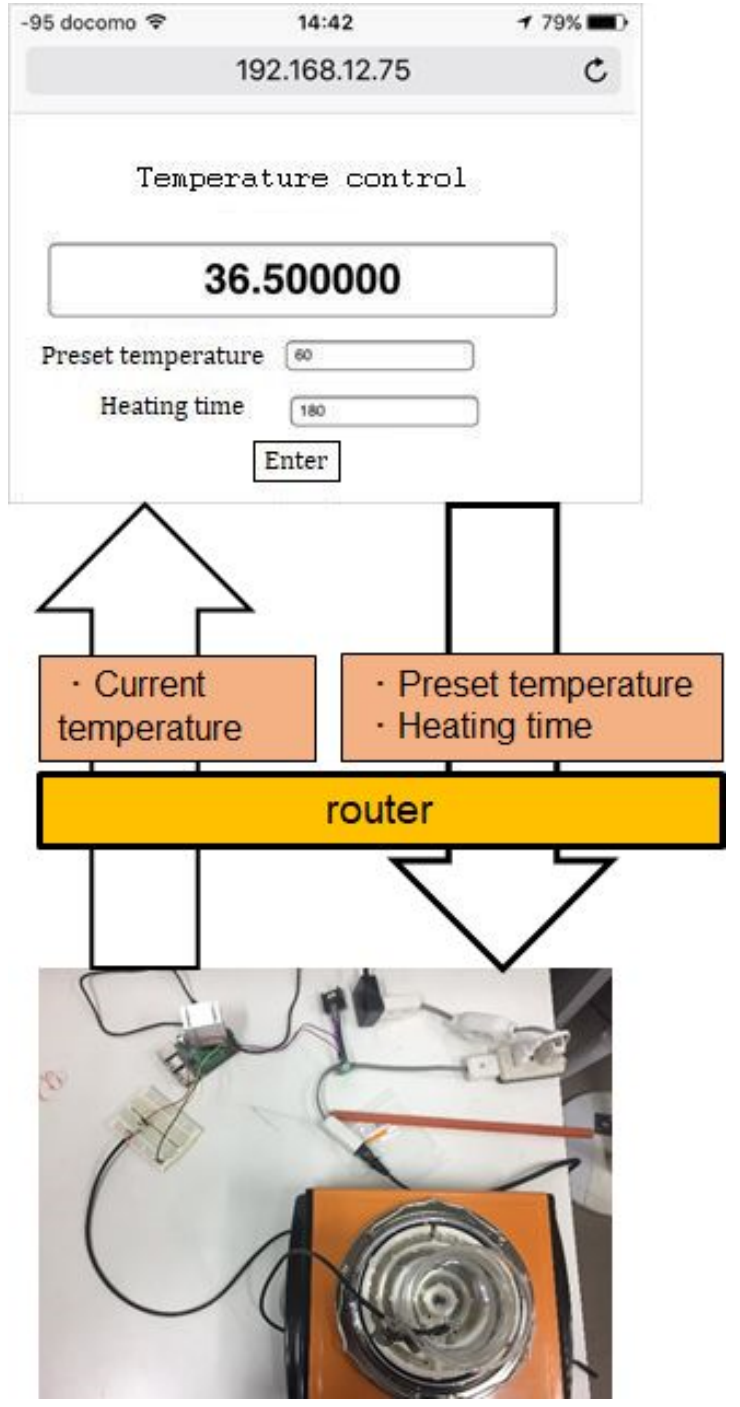

Fig.6 Data flow

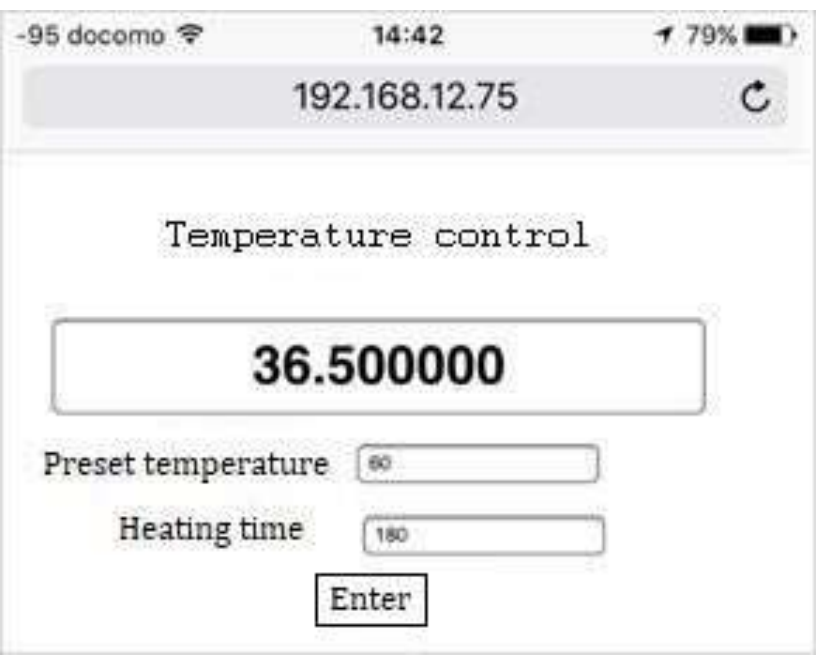

(a) Smartphone
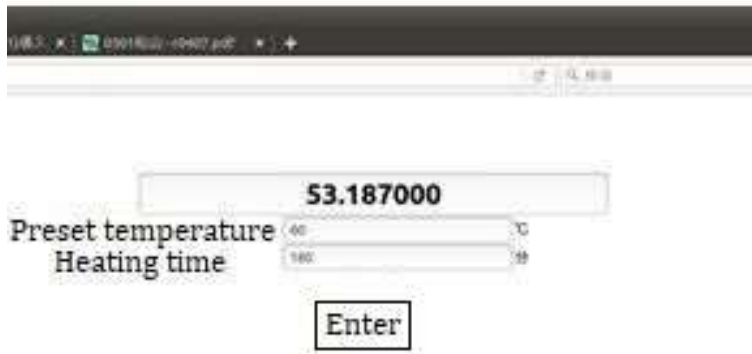

(b) PC

Fig.7 Image of application

Furthermore, because the connection to the control computer is carried out through a router, it can be accessed not only via the local network but also via Internet if we use global IP address.

The GUI application for control has plural methods including android application, iOS application, the PC software and so on, but in this time we choose an application that is mounted on the html page in order to be able to use any Internet terminal including a browser.

When you access these pages, preset ID and password are to be requested.

\section{Conclusions}

We have made a low temperature control unit which is cheaper and easy to use for small businesses by using IoT control system. It has been possible to conduct temperature control by transmitting data such as temperature setting or time setting to micro PC from a network terminal and displaying current setting or temperature to a network 
terminal from micro PC.

The improvement of safety function such as preventing illegal accesses or transmitting fault conditions is necessary for further research.

\section{References}

(1) https://www.raspberrypi.org

(2) https://archive.org/details/Raspberry_Pi_The_Complet e_Manual_7th_Edition

(3) https://fieldguide.gizmodo.com/the-beginners-guide-tothe-raspberry-pi-1782608097

(4) http://deviceplus.jp/hobby/raspberrypi_entry_034 